\title{
Plasma Cystatin C and High-Density Lipoprotein Are Important Biomarkers of Alzheimer's Disease and Vascular Dementia: A Cross-Sectional Study
}

\author{
Rui Wang ${ }^{1 \dagger}$, Zhaoyu Chen ${ }^{1 \dagger}$, Yongmei Fu ${ }^{2 \dagger}$, Xiaobo Wei ${ }^{1 \dagger}$, Jinchi Liao ${ }^{1}, \mathrm{Xu} \mathrm{Liu}^{1}$, \\ Bingjun $\mathrm{He}^{3}$, Yunqi Xu ${ }^{4}$, Jing Zou ${ }^{1}$, Xiaoyan Yang ${ }^{2}$, Ruihui Weng ${ }^{1}$, Sheng Tan $^{5}$, \\ Christopher McElroy ${ }^{6}$, Kunlin Jin ${ }^{6 *}$ and Qing Wang ${ }^{1,7 *}$
}

${ }^{1}$ Departments of Neurology, The Third Affiliated Hospital of Sun Yat-Sen University, Guangzhou, China, ${ }^{2}$ Departments of Emergency, The Third Affiliated Hospital of Sun Yat-Sen University, Guangzhou, China, ${ }^{3}$ Departments of Radiology, The Third Affiliated Hospital of Sun Yat-Sen University, Guangzhou, China, ${ }^{4}$ Department of Neurology, Nanfang Hospital, Southern Medical University, Guangzhou, China, ${ }^{5}$ Departments of Neurology, Zhujiang Hospital, Southern Medical University, Guangzhou, China, ${ }^{6}$ Department of Pharmacology and Neuroscience, University of North Texas Health Science Center, Fort Worth, TX, USA, ' ${ }^{7}$ Guangdong Province Key Laboratory of Brain Function and Disease, Guangzhou, China

OPEN ACCESS

Edited by:

Hanting Zhang,

West Virginia University, USA

Reviewed by: Ramesh Kandimalla,

Texas Tech University, USA

Penghua Wang,

New York Medical College, USA

${ }^{*}$ Correspondence: Kunlin Jin

Kunlin.Jin@unthsc.edu Qing Wang

denniswq@yahoo.com

${ }^{\dagger}$ These authors have contributed equally to this work.

Received: 18 October 2016 Accepted: 27 January 2017 Published: 07 February 2017

Citation:

Wang $R$, Chen Z, Fu Y, Wei X, Liao J, Liu X, He B, Xu Y, Zou J, Yang $X$, Weng $R$, Tan S, McElroy $C$, Jin K and Wang Q (2017) Plasma Cystatin C and High-Density Lipoprotein Are Important Biomarkers of Alzheimer's Disease and Vascular Dementia: A Cross-Sectional Study.

Front. Aging Neurosci. 9:26. doi: 10.3389/fnagi.2017.00026
Objectives: Cystatin C (Cys C) and high-density lipoprotein (HDL) play critical roles in neurodegenerative diseases, such as dementia, Alzheimer's disease (AD) and vascular dementia (VaD). However, whether they can be used as reliable biomarkers to distinguish patients with dementia from healthy subjects and to determine disease severity remain largely unknown.

Methods: We conducted a cross-sectional study to determine plasma Cys C and HDL levels of 88 patients with dementia (43 AD patients, $45 \mathrm{VaD}$ patients) and 45 healthy age-matched controls. The severity of dementia was determined based on the Schwab and England Activities of Daily Living (ADL) Scale, the Mini-mental State Examination (MMSE), the Global Deterioration Scale (GDS), the Lawton Instrumental ADL (IADL) Scale, and the Hachinski Ischemia Scale (Hachinski). Receiver operating characteristic $(\mathrm{ROC})$ curves were calculated to determine the diagnostic accuracy of Cys $\mathrm{C}$ and $\mathrm{HDL}$ levels in distinguishing patients with dementia from healthy subjects.

Results: We found that plasma Cys C levels were higher, but HDL levels were lower in $\mathrm{AD}$ and $\mathrm{VaD}$ patients respectively, compared to healthy control subjects. Yet, Cys $\mathrm{C}$ levels were highest among patients with VaD. Interestingly, plasma Cys $\mathrm{C}$ levels were significantly correlated with IADL Scale scores. In addition, the ROC curves for Cys C

\footnotetext{
Abbreviations: AD, Alzheimer's disease; ADL, Schwab and England Activities of Daily Living Scale; AUC, area under the curve; BUN, blood urea nitrogen; CNS, central nervous system; Cr, creatinine; Cys C, cystatin C; GDS, Global Deterioration Scale; Hachinski, Hachinski Ischemia Scale; HDL, high-density lipoprotein; H\&Y, modified Hoehn and Yahr Staging Scale; IADL, Lawton Instrumental Activities of Daily Living Scale; MMSE, Mini-mental State Examination; NINCDS-ADRDA, National Institute of Neurological and Communicative Diseases and Stroke-Alzheimer's Disease and Related Disorders Association; NINDS-AIREN, National Institute of Neurological Disorders and Stroke-Association Internationale pour la Recherche et l'Enseignement en Neurosciences; ROC, receiver operating characteristic; $r_{\mathrm{s}}$, Spearman's rank correlation coefficient; $r_{\mathrm{p}}$, Pearson's correlation coefficient; SD, standard deviation; UA, uric acid; VaD, vascular dementia.
} 
(area under the curve, AUC 0.816 for AD, AUC 0.841 for $\mathrm{VaD}$ ) and HDL (AUC 0.800 for $A D, A \cup C 0.731$ for $V a D$ ) exhibited potential diagnostic value in distinguishing $A D / N a D$ patients from healthy subjects. While the ROC curve for the combination of Cys $C$ and HDL (AUC 0.873 for AD, AUC 0.897 for $\mathrm{VaD}$ ) showed higher diagnostic accuracy in distinguishing $\mathrm{AD} / \mathrm{VaD}$ patients from healthy subjects than the separate curves for each parameter.

Conclusions: Our findings suggest that the inflammatory mediators Cys C and HDL may play important roles in the pathogenesis of dementia, and plasma Cys $\mathrm{C}$ and $\mathrm{HDL}$ levels may be useful screening tools for differentiating $A D / N a D$ patients from healthy subjects.

\section{Keywords: Alzheimer's disease, vascular dementia, cystatin C, high-density lipoprotein, dementia}

\section{Highlights}

- Plasma Cys C levels were higher in patients with $\mathrm{AD} / \mathrm{VaD}$ than in healthy subjects.

- Plasma HDL levels were lower in patients with $\mathrm{AD} / \mathrm{VaD}$ than in healthy subjects.

- Plasma Cys C levels were significantly correlated with dementia.

- The ROC curve for the combination of Cys C and HDL showed potential diagnostic value in distinguishing $\mathrm{AD} / \mathrm{VaD}$ from healthy subjects.

\section{INTRODUCTION}

Dementia represents a broad category of brain diseases that usually cause declines in memory and gradual decreases in cognition that affect a person's quality of life (Paulsen et al., 2013). Common types of dementia include Alzheimer's disease $(\mathrm{AD})$, vascular dementia $(\mathrm{VaD})$, Lewy body dementia (LBD), frontotemporal dementia (FTD), multiple system atrophy dementia (MSA-D) and Parkinson's disease dementia (PDD). Among these, $\mathrm{AD}$ and $\mathrm{VaD}$ are the most common types (PosadaDuque et al., 2014; $\mathrm{Xu}$ et al., 2014). AD is characterized by the accumulation of amyloid- $\beta$ (A $\beta$ ) peptides and Tau (Kandimalla et al., 2011, 2014; Bourdenx et al., 2015; Hoppe et al., 2015). Meanwhile, $\mathrm{VaD}$ is characterized by the occurrence of minor strokes and the development of cognitive deficits (Ray et al., 2013). However, it is hard to differentiate from each other only based on the clinical features (Ray et al., 2013).

The cystatins (Cyss) are a family of cysteine protease inhibitors. Cys B and C have been increasingly investigated in neurological diseases such as $\mathrm{AD}$ and $\mathrm{VaD}$. Cys $\mathrm{B}$, a member of the cysteine protease inhibitor family, plays an important role in regulating abnormal accumulation of $A \beta$ peptide and maintaining lipofuscin-related autofluorescence and giant lipidcontaining autolysosomes in AD (Yang et al., 2011a,b, 2014; Boyle et al., 2013). Cys C, a potent cysteine inhibitor that is encoded by the Cys C (CST3) gene and that is secreted by all human tissues, is found in all body fluids (Heywood et al., 2015). It has been used as a biomarker of renal function as well as a strong predictor of $\mathrm{VaD}$ and $\mathrm{AD}$ (Kaur and Levy, 2012;
Zhong et al., 2013). Multiple lines of evidence indicate that Cys $\mathrm{C}$ is functionally associated with anti-inflammation (Dutta et al., 2012; Jonsdottir et al., 2013) and exerts protective effects against age-related diseases (Xu et al., 2005; Mi et al., 2007; Kaur et al., 2010; Liu et al., 2014).

In $\mathrm{AD}$ and $\mathrm{VaD}$, lipoproteins play crucial roles in preserving cognitive function (Ray et al., 2013; Ahmed et al., 2014; Dias et al., 2014). High-density lipoprotein (HDL) is part of a heterogeneous group of lipoprotein particles that exist in the systemic circulation and brain and mainly facilitate the clearance and delivery of lipids and lipid-related molecules from and throughout the body, respectively (Hottman et al., 2014). Several lines of evidence suggest that plasma HDL and its main protein component, apoA-I, also possess potent vasoprotective properties such as facilitating improvements in vascular function, inhibiting inflammation, suppressing endothelial reparation, preventing lipid oxidation, and stimulating endothelial repair (Stukas et al., 2014).

Both Cys C and HDL not only modulate dementia but also associate with vascular function and mediate vasorelaxation, inflammation, and oxidative stress (Dutta et al., 2012; Jonsdottir et al., 2013; Stukas et al., 2014). Therefore, we are interested in investigating the levels of Cys C and HDL in different types of dementia. To our knowledge there is a paucity of studies exploring the combined effects of Cys $\mathrm{C}$ and HDL in patients with dementia. In this study, we investigate whether Cys $\mathrm{C}$ and HDL are associated with the severity and prevalence of different types of dementia. The primary aim of this study was to compare plasma Cys C/HDL levels between patients with $\mathrm{AD} / \mathrm{VaD}$ and healthy subjects. The secondary aim of this study was to explore the diagnostic value of plasma Cys C/HDL in dementia. Lastly, we also aimed to determine the correlations between the above plasma markers and the severity of dementia.

\section{MATERIALS AND METHODS}

\section{Patients and Ethics Statement}

This cross-sectional study was performed at the Department of Neurology of the Third Affiliated Hospital Sun Yat-sen University, Guangzhou, China. From November 2012 to October 2015, a total of 88 patients with dementia ( $A D$ and $\mathrm{VaD}$ ) 
were recruited for this study. Additionally, 43 patients with $\mathrm{AD}$ (20 males and 23 females) were enrolled, and their diagnoses were confirmed using the National Institute of Neurological and Communicative Diseases and Stroke-AD and Related Disorders Association (NINCDS-ADRDA) criteria for AD (McKhann et al., 1984). This study also included 45 patients with $\mathrm{VaD}$ (24 males and 21 females) whose diagnoses were confirmed using the National Institute of Neurological Disorders and StrokeAssociation Internationale pour la Recherche et l'Enseignement en Neurosciences (NINDS-AIREN) criteria for $\mathrm{VaD}$ (Román et al., 1993). The MMSE scores of all patients were less than 25. A total of 45 healthy age-matched subjects (16 males and 29 females) were recruited from the outpatient setting and served as the control group. The control group was selected from the Medical Examination Centre of the Third Affiliated Hospital of Sun Yat-sen University. In this study, no subject presented with hypertension, cardiopathy, diabetes or renal dysfunction. In addition, none of the patients presented abnormal levels of the prostate carcinoma-related mediators prostate-specific antigen (PSA), carcinoembryonic antigen (CEA), and alpha-fetoprotein (AFP).

This study was approved by the ethics committee of the Third Affiliated Hospital of Sun Yat-sen University and was conducted according to the principles outlined in the revised Declaration of Helsinki of 1975 and the National Institutes of Health Human Subjects Policies and Guidelines released in 1999. All participants provided written consent to participate in the investigation and allowed researchers to measure their plasma Cys $\mathrm{C}$ and HDL levels. All subjects participated in the following standardized assessments: the Schwab and England Activities of Daily Living (ADL) Scale, the Webster Scale, the modified Hoehn and Yahr Staging Scale (H\&Y), the Mini-mental State Examination (MMSE), the Global Deterioration Scale (GDS), the Lawton Instrumental ADL (IADL) Scale, and the Hachinski Ischemia Scale (Hachinski). Patients with different types of disease participated in different standardized assessments, which were all conducted in a blinded manner.

\section{Study Design}

Experienced neurologists were recruited to perform evaluations and complete neurological examinations of both the inpatients and the outpatients. All patients with AD met the NINCDSADRDA criteria, and all patients with $\mathrm{VaD}$ met the NINDSAIREN criteria (Román et al., 1993). The following patients were excluded: (1) patients with physical disability due to neurological disorders other than $\mathrm{AD}$ or $\mathrm{VaD}$ such as congenital diseases or psychosis; (2) patients with somatic disabilities caused by trauma or other diseases that may affect ADL and IADL, including pain syndromes, advanced diabetes mellitus, malignancy, renal failure, hepatic failure, cardiopathy, severe anemia, or any other acute or chronic debilitating or life-threatening disease/state; (3) patients with MMSE scores higher than 25 (25 was included in the study); and (4) patients who refused to participate in the study. All subjects completed the following standardized assessments: the ADL Scale, IADL Scale and GDS, which stratified patients based on their clinical disabilities (Kaufman et al., 2013); Hachinski, which stratified patients based on whether they had $\mathrm{VaD}, \mathrm{AD}$ or another type of dementia $(\mathrm{AD}<4, \mathrm{VaD}>7)$; and the MMSE, which evaluated patient cognitive abilities. All scales were available and validated for the Chinese population. All subjects have been scanned by magnetic resonance imaging (MRI), and the typical MRI for normal control, $\mathrm{AD}$ and $\mathrm{VaD}$ patients are shown in Figure 1.

\section{Blood Sample Measurements}

Five milliliters of venous blood was taken to measure Cys $\mathrm{C}$ and HDL levels. All measurements were repeated three times. Plasma was isolated by centrifugation at $3000 \mathrm{rpm}$ for $10 \mathrm{~min}$ within $1 \mathrm{~h}$ of sample collection. The separated plasma was stored at $-30^{\circ} \mathrm{C}$

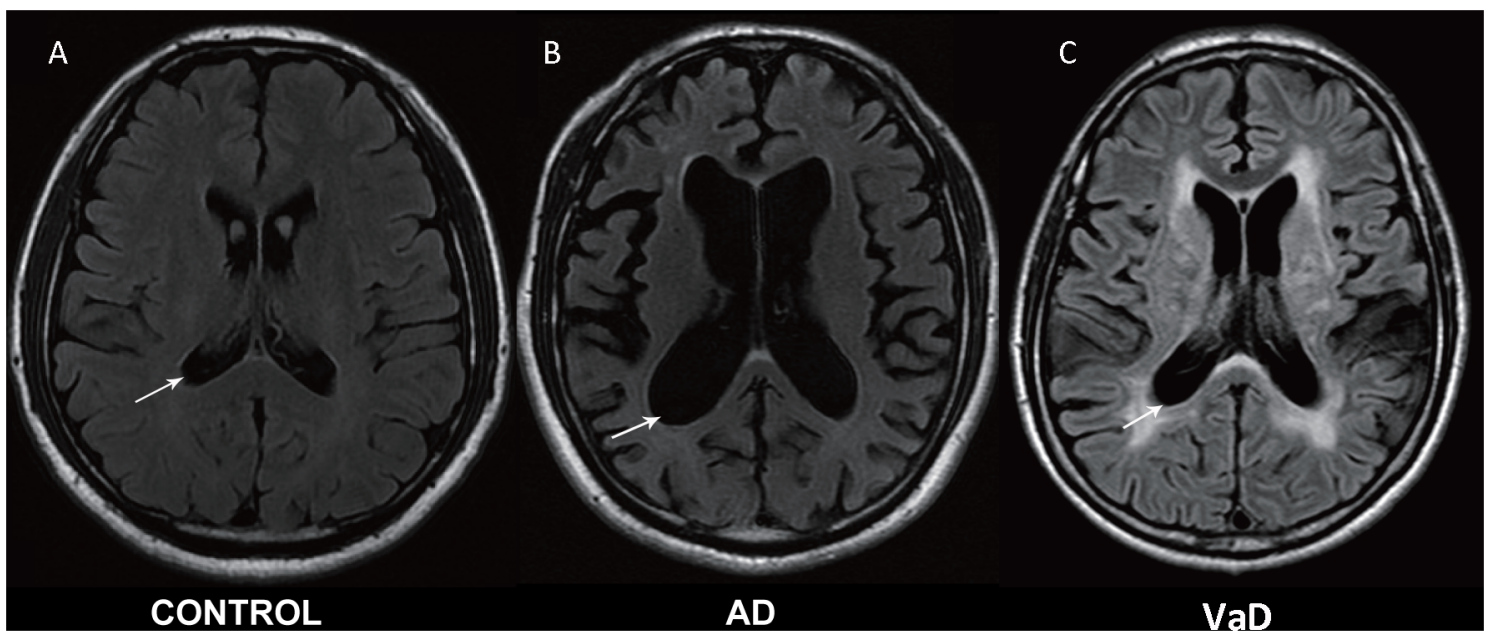

FIGURE 1 | Magnetic resonance imaging (MRI) images in normal control, Alzheimer's disease (AD) and vascular dementia (VaD) patients. (A) Healthy control. (B) Cerebral atrophy and enlargement of the cerebral lateral ventricles of AD patients. (C) Leukoencephalopathy and encephalomalacia foci of VaD patients. All MRI are shown in T2-weighted image. 
before laboratory evaluation (Chen et al., 2015; Gao et al., 2015). Cys $\mathrm{C}$ levels were measured with a latex-enhanced reagent $(\mathrm{N}$ Latex Cys C, Dade Behring, Deerfield, IL, USA), using a Behring BN ProSpec Analyzer (Dade Behring). Plasma HDL levels were measured via direct enzymatic methods, using commercial kits (Cholestest N-HDL, Sekisui Medical, Tokyo, Japan) with less than $3 \%$ intra-assay and inter-assay coefficients of variance.

\section{Statistical Analysis}

All continuous variables (age; MMSE, ADL, IADL, and GDS scores; and HDL and Cys C levels) were presented as the mean \pm standard deviation if the data were normally distributed or as medians ( $\min , \max$ ) if the data were not normally distributed. Categorical variables are presented as percentages. ADL and IADL scores were determined by summing individual items. To assess the significance of differences between groups, Student's $t$ test was applied when the data were normally distributed. Tukey's post hoc analysis was conducted to compare differences in HDL and Cys C levels among normal subjects, according to gender. Pearson's correlation $\left(r_{\mathrm{p}}\right)$ and Spearman's rank correlation $\left(r_{\mathrm{s}}\right)$ coefficient were used to evaluate correlations between different clinical parameters for normal data and non-normal data, respectively. Receiver operating characteristic (ROC) analysis was conducted to assess the diagnostic value of specific clinical biomarkers (Cys C, HDL) with respect to identifying the abovementioned diseases. In addition, an ROC curve for the combination of Cys $\mathrm{C}$ and HDL was calculated via logistic regression analysis to determine the value of this combination in predicting disease progression. $p$ values $<0.05$ were deemed statistically significant, and SPSS 13.0 software (Chicago, IL, USA) was used for the statistical analyses.

\section{RESULTS}

\section{Patient Characteristics}

This cross-sectional study included $43 \mathrm{AD}$ patients (20 males [47\%] and 23 [53\%] females), $45 \mathrm{VaD}$ patients (24 males [53\%] and 21 [47\%] females), and 45 healthy subjects (16 males [36\%] and 29 females [64\%]). The mean ages of the $\mathrm{AD}$ patients, VaD patients and normal controls were $67.35 \pm 10.48$, $69.11 \pm 7.98$ and $64 \pm 6.47$ years, respectively. Clinical evaluating parameters in $\mathrm{AD}$ and $\mathrm{VaD}$ were shown in Tables 1A. There was no significance difference in age between the patients and control subjects (AD vs. Control, $p=1.000$; VaD vs. Control, $p=0.191$; Student's $t$-test). The demographic and clinical data for the subjects are shown in Tables 1A,B).

\section{Comparisons of Cys C/HDL Levels between Patients with Dementia and Healthy Subjects}

In this study, significant differences in plasma Cys $\mathrm{C}$ levels were observed between patients with dementia $(\mathrm{AD} / \mathrm{VaD})$ and healthy subjects (Table 2). Plasma Cys $\mathrm{C}$ levels were higher in $\mathrm{AD}$ and $\mathrm{VaD}$ patients than in normal subjects $(\mathrm{AD}, 1.03 \pm 1.94$ vs. $0.83 \pm 0.13,{ }^{* *} p<0.001 ; \mathrm{VaD}, 1.07 \pm 0.23$ vs. $0.83 \pm 0.13$,
${ }^{* *} p<0.001$; Student's $t$-test, (Tables 1A,B; Figure 2). Moreover, in patients with $\mathrm{AD}$ or $\mathrm{VaD}$, HDL levels were significantly lower than in healthy subjects $(\mathrm{AD}: 1.15 \pm 0.32$ vs. $1.42 \pm 0.25$, ${ }^{*} p=0.023 ; \mathrm{VaD}: 1.09 \pm 0.32$ vs. $1.42 \pm 0.25,{ }^{* *} p=0.001$, Student's $t$-test, Table 1B; Figure 2).

\section{Correlations between Cys $C$ and HDL Levels and MMSE, ADL, IADL and GDS Scores}

We utilized correlation analysis to investigate the correlations between the abovementioned inflammatory mediators and various assessment tools. Depending on the data distribution, Pearson's and Spearman's correlations were used separately for different diseases to evaluate the correlations between disease severity and clinical variables (Tables $\mathbf{3 A}, \mathbf{B})$. In $\mathrm{AD}$ patients, there were significant correlations between Cys $C$ levels and Hachinski scores $\left(r_{\mathrm{s}}=0.349,{ }^{*} p=0.022\right.$, Table 3A) and Cys C levels and age $\left(r_{s}=0.575,{ }^{* *} p=0.000\right.$, Table 3A $)$. However, there were no significant correlations between Cys C/HDL levels and the scores of the other assessments. In $\mathrm{VaD}$ patients, a significant correlation was noted only between HDL and age $\left(r_{\mathrm{s}}=0.403\right.$, ${ }^{* *} p=0.006$, Table 3B).

To eliminate the influence of confounders (e.g., gender), we divided the $\mathrm{AD}$ and $\mathrm{VaD}$ patients and the healthy subjects into two groups (females/males). In male $\mathrm{AD}$ patients, there were significant correlations between Cys C levels and age $\left(r_{\mathrm{s}}=0.619\right.$, ${ }^{* *} p=0.005$, Table 3A) and Cys $C$ levels and IADL scores $\left(r_{\mathrm{s}}=-0.464,{ }^{*} p=0.046\right.$, Table 3A). Meanwhile, in female AD patients, there were significant correlations between Cys $\mathrm{C}$ levels and MMSE scores $\left(r_{\mathrm{s}}=-0.413,{ }^{*} p=0.045\right.$, Table 3A) Cys C levels and Hachinski scores $\left(r_{s}=0.524,{ }^{* *} p=0.009\right.$, Table 3A), and Cys $C$ levels and age $\left(r_{\mathrm{s}}=0.540,{ }^{* *} p=0.006\right.$, Table 3A). However, in $\mathrm{VaD}$ patients, a significant correlation was observed only between HDL and age $\left(r_{\mathrm{s}}=0.552,{ }^{* *} p=0.009\right.$, Table 3B).

\section{ROC Analysis of the Utility of Cys C and HDL Levels in the Diagnosis of Dementia}

ROC analysis was conducted to determine the diagnostic value of Cys $\mathrm{C}$ and $\mathrm{HDL}$, according to the area under the ROC curve (AUC). Using ROC analysis, we determined the capacity of the above markers to distinguish patients with dementia from normal subjects. We also analyzed Cys $\mathrm{C}$ and HDL levels in $\mathrm{AD}$ patients. The AUC for Cys C was 0.816 (95\% CI: 0.724-0.908, $\left.{ }^{* * *} p<0.001\right)$; the cut-off was $0.91 \mu \mathrm{mol} / \mathrm{L}$, with a sensitivity of $79 \%$ and a specificity of $78 \%$. Similarly, the AUC for HDL was $0.731 \mu \mathrm{mol} / \mathrm{L}$ (95\% CI: $\left.0.759-0.923,{ }^{* * *} p<0.001\right)$; the cut-off was $1.04 \mu \mathrm{mol} / \mathrm{L}$, with a sensitivity of $42 \%$ and a specificity of $98 \%$. Furthermore, in $\mathrm{VaD}$ patients, the AUC for Cys C was 0.841 (95\% CI: $0.627-0.835,{ }^{* * *} p<0.001$ ); the cut-off was at $0.97 \mu \mathrm{mol} / \mathrm{L}$, with a sensitivity of $81 \%$ and a specificity of $51 \%$. The AUC for Cys C was 0.800 (95\% CI: 0.705-0.894, $\left.{ }^{* * *} p<0.001\right)$; the cut-off was $1.14 \mu \mathrm{mol} / \mathrm{L}$, with a sensitivity of $64 \%$ and a specificity of $89 \%$ (Figure 3 ).

In addition, we also conducted an ROC analysis for the combination of Cys $\mathrm{C}$ and HDL in different types of dementia. The AUC for AD was 0.873 (95\% CI: $0.797-0.950,{ }^{* * *} p<0.001$ ), 
TABLE 1A | Clinical parameters in Alzheimer's disease (AD).

\begin{tabular}{|c|c|c|c|c|c|c|c|c|c|}
\hline \multirow[t]{2}{*}{ Variable } & \multicolumn{3}{|c|}{$A D$} & \multicolumn{3}{|c|}{ AD M } & \multicolumn{3}{|c|}{$A D F$} \\
\hline & Mean (SD) & Min & Max & Mean (SD) & Min & Max & Mean (SD) & Min & Max \\
\hline MMSE & 14.00 & 0 & 22 & 14.00 & 0 & 22 & 12.50 & 0 & 21 \\
\hline GDS & 5.00 & 3 & 7 & 5.00 & 3 & 7 & 5.00 & 3 & 6 \\
\hline Hachinski & 2.00 & 1 & 3 & 2.00 & 1 & 3 & 2.00 & 1 & 3 \\
\hline IADL & 5.00 & 0 & 17 & 5.00 & 0 & 15 & 9.00 & 0 & 17 \\
\hline
\end{tabular}

Abbreviations: MMSE, Mini-mental State Examination; ADL, Schwab and England Activities of Daily Living Scale; GDS, Global Deterioration Scale; Hachinski, Hachinski Ischemia Scale; IADL, Lawton Activities of Daily Living Scale.

TABLE 1B | Clinical parameters in vascular dementia (VaD).

\begin{tabular}{|c|c|c|c|c|c|c|c|c|c|}
\hline \multirow[t]{2}{*}{ Variable } & \multicolumn{3}{|c|}{ VaD } & \multicolumn{3}{|c|}{ VaD M } & \multicolumn{3}{|c|}{ VaD F } \\
\hline & Mean (SD) & Min & Max & Mean (SD) & Min & Max & Mean (SD) & Min & Max \\
\hline MMSE & $16.10(5.18)$ & 3 & 24 & $15.29(5.20)$ & 3 & 23 & $17.19(5.10)$ & 6 & 24 \\
\hline GDS & 4.00 & 2 & 6 & 4.00 & 3 & 5 & 4.00 & 2 & 6 \\
\hline Hachinski & 11.00 & 7 & 14 & 9.50 & 7 & 14 & 11.00 & 7 & 13 \\
\hline IADL & $13.13(3.50)$ & 6 & 21 & $13.13(3.44)$ & 6 & 21 & 13.38 & 4 & 19 \\
\hline
\end{tabular}

Abbreviations: MMSE, Mini-mental State Examination; ADL, Schwab and England Activities of Daily Living Scale; GDS, Global Deterioration Scale; Hachinski, Hachinski Ischemia Scale; IADL, Lawton Activities of Daily Living Scale.

with a sensitivity of $91 \%$ and a specificity of $73 \%$, and the cut-off was 0.36 , depending on the predicted risk algorithm. Meanwhile, the AUC for $\mathrm{VaD}$ was 0.897 (95\% CI: $0.831-0.964,{ }^{* * *} p<0.001$ ); the cut-off was 0.34 , depending on the predicted risk algorithm, with a sensitivity of $91 \%$ and a specificity of $78 \%$. These data suggest that the ROC curve for the combination of $\mathrm{AD} / \mathrm{VaD}$ has a higher diagnostic value than the separate curves for uric acid (UA) or $\mathrm{HDL}$ alone in distinguishing $\mathrm{AD}$ and $\mathrm{VaD}$ patients from healthy subjects.

\section{DISCUSSION}

In the present study, we explored variations in plasma Cys C and $\mathrm{HDL}$ levels in $\mathrm{AD}$ and $\mathrm{VaD}$ and noted several interesting results. First, we observed a remarkable increase in plasma Cys $\mathrm{C}$ levels and a decrease in HDL levels in $\mathrm{AD} / \mathrm{VaD}$ patients compared to healthy subjects. Second, we noted significant correlations between plasma Cys C levels and severity scores in $\mathrm{AD}$ and $\mathrm{VaD}$ patients. Third, ROC analysis strongly suggested that the combination of Cys $\mathrm{C}$ and HDL can significantly distinguish $\mathrm{AD}$ and $\mathrm{VaD}$ patients from healthy subjects and can therefore be used as a new screening and diagnostic instrument.
The AUC increased after incorporating plasma Cys $\mathrm{C}$ and HDL levels. This means that the diagnostic accuracy of the combination of the two variables was superior to that of either variable alone in differentiating $\mathrm{AD} / \mathrm{VaD}$ patients from healthy subjects. To our knowledge, this is the first study to explore variations in plasma Cys $\mathrm{C}$ and $\mathrm{HDL}$ levels in $\mathrm{AD}$ and $\mathrm{VaD}$ patients.

$\mathrm{AD}$ and $\mathrm{VaD}$ are neurodegenerative diseases with different etiologies and pathogeneses. Therefore, it is important to explore the underlying mechanisms of these diseases and the usefulness of specific biomarkers in evaluating their severity and progression. There is evidence that inflammatory responses may be associated with the pathogenesis of $\mathrm{AD}$ and $\mathrm{VaD}$, and there are also some clinical similarities between $\mathrm{AD}$ and $\mathrm{VaD}$ as both diseases cause gradual impairments in cognition, follow a progressive course, and affect activities of daily living. Several lines of recent evidence show that both Cys C and HDL can act as anti-inflammatory mediators and exert neuroprotective effects against $\mathrm{AD}$ and $\mathrm{VaD}$ progression (Gauthier et al., 2011; Hottman et al., 2014; Stukas et al., 2014). However, whether Cys $\mathrm{C}$ and HDL can be used in the evaluation and diagnosis of $\mathrm{AD}$ and $\mathrm{VaD}$ remains unknown. Therefore, we examined

TABLE 2 | Comparison of Cystatin C (Cys C), high-density lipoprotein (HDL), CREAT, blood urea nitrogen (BUN) and uric acid (UA) levels among AD patients, VaD patients and normal healthy subjects.

\begin{tabular}{|c|c|c|c|c|c|}
\hline Variable & $\begin{array}{c}\text { Control } \\
\text { Mean } \pm \text { SD }\end{array}$ & $\begin{array}{c}\text { AD } \\
\text { Mean } \pm \text { SD }\end{array}$ & $\begin{array}{c}\text { VaD } \\
\text { Mean } \pm \text { SD }\end{array}$ & $\begin{array}{c}p \text { value } \\
\text { Control vs. AD }\end{array}$ & $\begin{array}{c}p \text { value } \\
\text { Control vs. VaD }\end{array}$ \\
\hline CYSC & $0.83 \pm 0.13$ & $1.03 \pm 1.94$ & $1.07 \pm 0.23$ & $<0.001^{* *}$ & $<0.001^{* *}$ \\
\hline $\mathrm{HDL}$ & $1.42 \pm 0.25$ & $1.15 \pm 0.32$ & $1.09 \pm 0.32$ & $0.023^{*}$ & $0.001^{* *}$ \\
\hline CREAT & $69.00 \pm 12.89$ & $75.67 \pm 13.76$ & $77.41 \pm 17.94$ & 0.558 & 0.257 \\
\hline BUN & $5.51 \pm 1.33$ & $5.57 \pm 1.61$ & $5.62 \pm 1.92$ & 1.000 & 1.000 \\
\hline UA & $316.64 \pm 59.28$ & $345.43 \pm 91.07$ & $323.31 \pm 93.84$ & 0.772 & 1.000 \\
\hline Age & $64 \pm 6.47$ & $67.35 \pm 10.48$ & $69.11 \pm 7.98$ & 1.000 & 0.191 \\
\hline
\end{tabular}

${ }^{*} p<0.05,{ }^{* *} p<0.01$. Student's t test. 

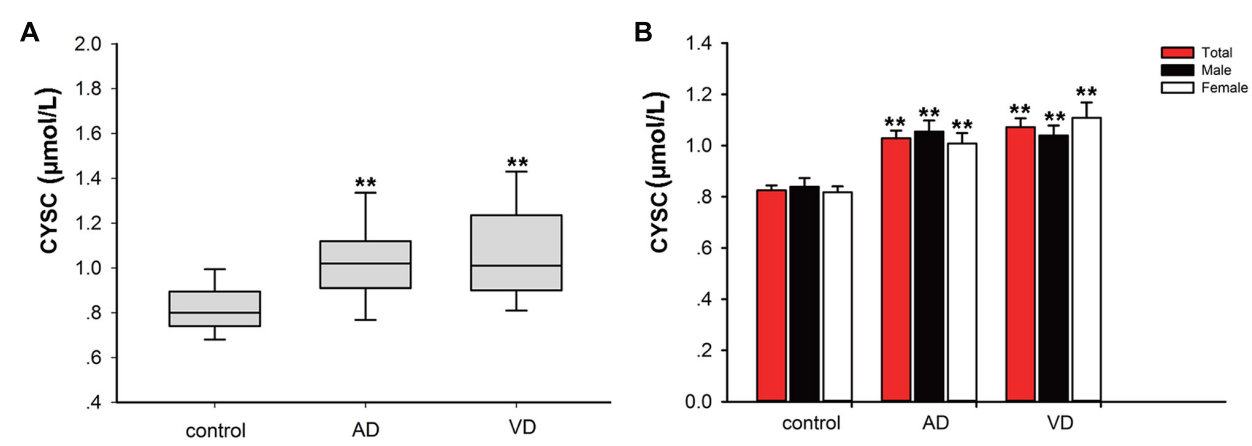

C

D
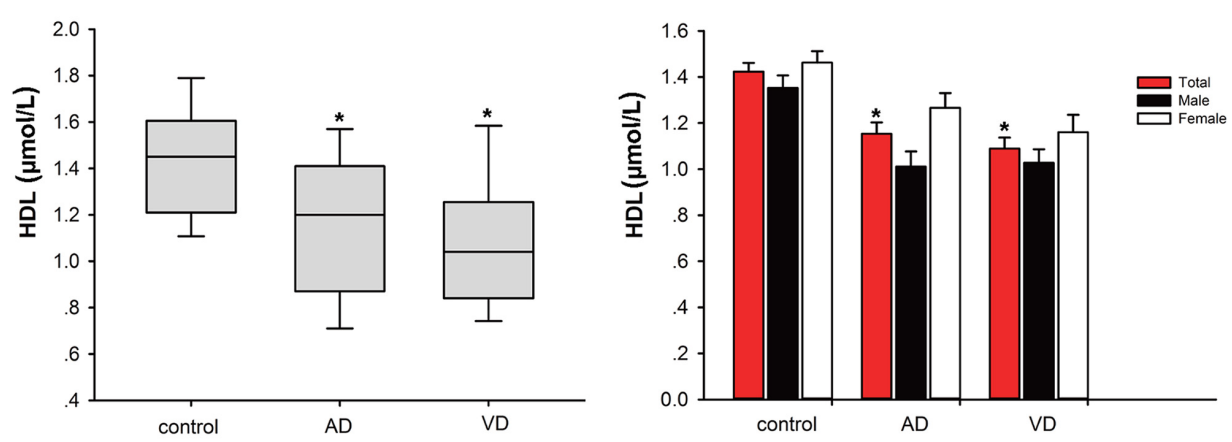

FIGURE 2 | Comparison of Cystatin C (Cys C) and high-density lipoprotein (HDL) levels between AD/VaD patients and control subjects.

(A) Comparison of Cys $\mathrm{C}$ levels between the dementia and control groups. ${ }^{* *} \mathrm{AD}$ vs. control, $p<0.001$; ${ }^{* *} \mathrm{VaD}$ vs. control, $p<0.001$. (B) Comparison of Cys $\mathrm{C}$ levels between the dementia and control groups according to gender. ${ }^{*} \mathrm{AD}$ (male) vs. control (male), $p=0.035 ;{ }^{* *} \mathrm{AD}$ (female) vs. control (female), $p=0.014 ;{ }^{* *} \mathrm{VaD}$ (male) vs. control (male), $p=0.043 ;{ }^{* *} \mathrm{VaD}$ (female) vs. control (female), $p<0.001$. (C) Comparison of HDL levels between the dementia and control groups. ${ }^{*} \mathrm{AD}$ vs. control, $p=0.023$; ** VaD vs. control, $p=0.001$. (D) Comparison of HDL levels between the dementia and control groups according to gender. No significant differences in HDL levels were found between the dementia (male) and control (male) and dementia (female) and control (female) groups.

TABLE 3A | Spearman's rank correlation coefficient $\left(r_{\mathrm{s}}\right)$ and $p$ values pertaining to the relationships between clinical variables and MMSE, IADL, GDS, Hachinski and ADL scores in AD.

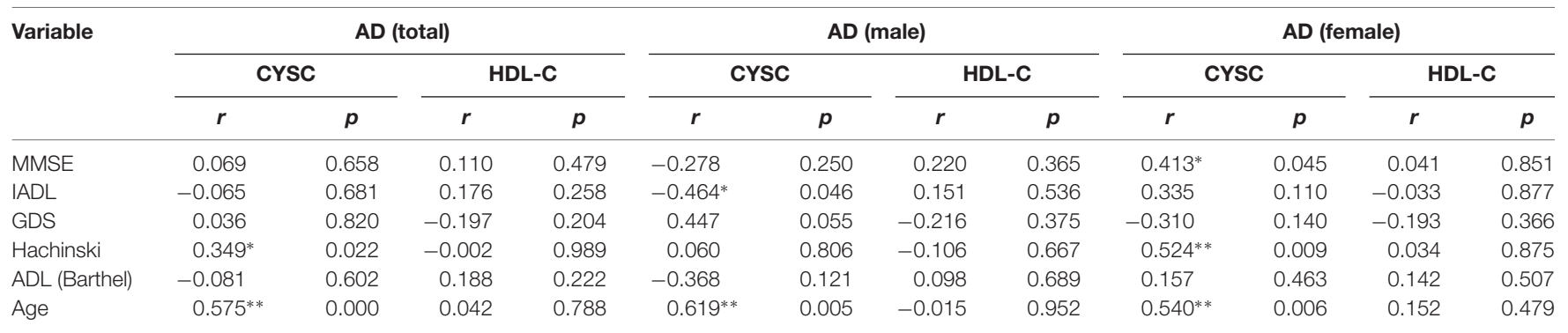

${ }^{*} p<0.05,{ }^{* *} p<0.01$. Abbreviations: $r$, Spearman's rank correlation coefficient; MMSE, Mini-mental State Examination; ADL, Schwab and England Activities of Daily Living Scale; GDS, Global Deterioration Scale; Hachinski, Hachinski Ischemia Scale; IADL, Lawton Activities of Daily Living Scale.

whether these anti-inflammatory mediators can be employed as viable and reliable biomarkers in diagnosing $\mathrm{AD}$ and $\mathrm{VaD}$ and in assessing their severities. We also identified clinical variables that are associated with $\mathrm{AD}$ and $\mathrm{VaD}$ in different gender groups.

Although the present, Cys C's function in the brain is unclear, but it has been implicated in both neuronal degeneration and nervous system repair. Enhanced CysC expression occurs in human patients and in animal models of neurodegenerative conditions (Liu et al., 2014). In our study, we noted significantly increased plasma Cys $\mathrm{C}$ levels in $\mathrm{AD}$ and $\mathrm{VaD}$ patients compared to healthy subjects. These results were consistent with previous reports. Similar results were observed in two previous crosssectional studies that noted higher and lower plasma Cys C levels in $\mathrm{AD}$ and $\mathrm{MCI}$ mild cognitive impairment (MCI) patients, respectively, and an increasing but non-significant trend in these levels in $\mathrm{AD}$ patients compared to those in healthy subjects (Ghidoni et al., 2010; Sundelöf et al., 2010). This finding implies that plasma Cys $\mathrm{C}$ may play an important role in $\mathrm{AD}$ and $\mathrm{VaD}$. However, different studies have reported lower CSF levels of Cys $\mathrm{C}$ in $\mathrm{AD}$ patients and no significant changes in plasma Cys $\mathrm{C}$ levels in $\mathrm{AD}$ patients compared to controls (Hansson et al., 2009; 
TABLE 3B | Pearson and Spearman's rank correlation coefficient $\left(r_{\mathrm{s}}\right)$ and $p$ values pertaining to the relationship between clinical variables and MMSE, IADL, GDS, Hachinski and ADL scores in VaD.

\begin{tabular}{|c|c|c|c|c|c|c|c|c|c|c|c|c|}
\hline \multirow[t]{3}{*}{ Variable } & \multicolumn{4}{|c|}{ VaD (total) } & \multicolumn{4}{|c|}{ VaD (male) } & \multicolumn{4}{|c|}{ VaD (female) } \\
\hline & \multicolumn{2}{|c|}{ CYSC } & \multicolumn{2}{|c|}{ HDL-C } & \multicolumn{2}{|c|}{ CYSC } & \multicolumn{2}{|c|}{ HDL-C } & \multicolumn{2}{|c|}{ CYSC } & \multicolumn{2}{|c|}{ HDL-C } \\
\hline & $r$ & $p$ & $r$ & $p$ & $r$ & $p$ & $r$ & $p$ & $r$ & $p$ & $r$ & $p$ \\
\hline MMSE & 0.036 & 0.816 & 0.150 & 0.326 & -0.132 & 0.538 & 0.085 & 0.694 & 0.121 & 0.601 & 0.147 & 0.525 \\
\hline IADL & -0.028 & 0.854 & 0.045 & 0.781 & 0.014 & 0.948 & 0.195 & 0.361 & -0.042 & 0.858 & -0.246 & 0.282 \\
\hline GDS & -0.003 & 0.986 & 0.166 & 0.277 & 0.082 & 0.704 & 0.168 & 0.433 & -0.010 & 0.967 & 0.199 & 0.388 \\
\hline Hachinski & 0.121 & 0.430 & -0.074 & 0.631 & 0.071 & 0.742 & -0.133 & 0.537 & 0.216 & 0.348 & -0.116 & 0.617 \\
\hline ADL (Barthel) & 0.054 & 0.724 & -0.016 & 0.919 & 0.149 & 0.487 & 0.236 & 0.267 & -0.022 & 0.926 & -0.178 & 0.440 \\
\hline Age & 0.128 & 0.401 & $0.403^{* *}$ & 0.006 & -0.014 & 0.949 & 0.195 & 0.361 & 0.317 & 0.161 & $0.552^{* *}$ & 0.009 \\
\hline
\end{tabular}

${ }^{*} p<0.05,{ }^{*} p<0.01$. Abbreviations: MMSE and IADL scale scores were analyzed via Pearson's correlation; GDS, ADL scale scores and Hachinski scores were analyzed by Spearman's rank correlation; MMSE, Mini-mental State Examination; ADL, Schwab and England Activities of Daily Living Scale; GDS, Global Deterioration Scale; Hachinski, Hachinski Ischemia Scale; IADL, Lawton Instrumental Activities of Daily Living Scale.
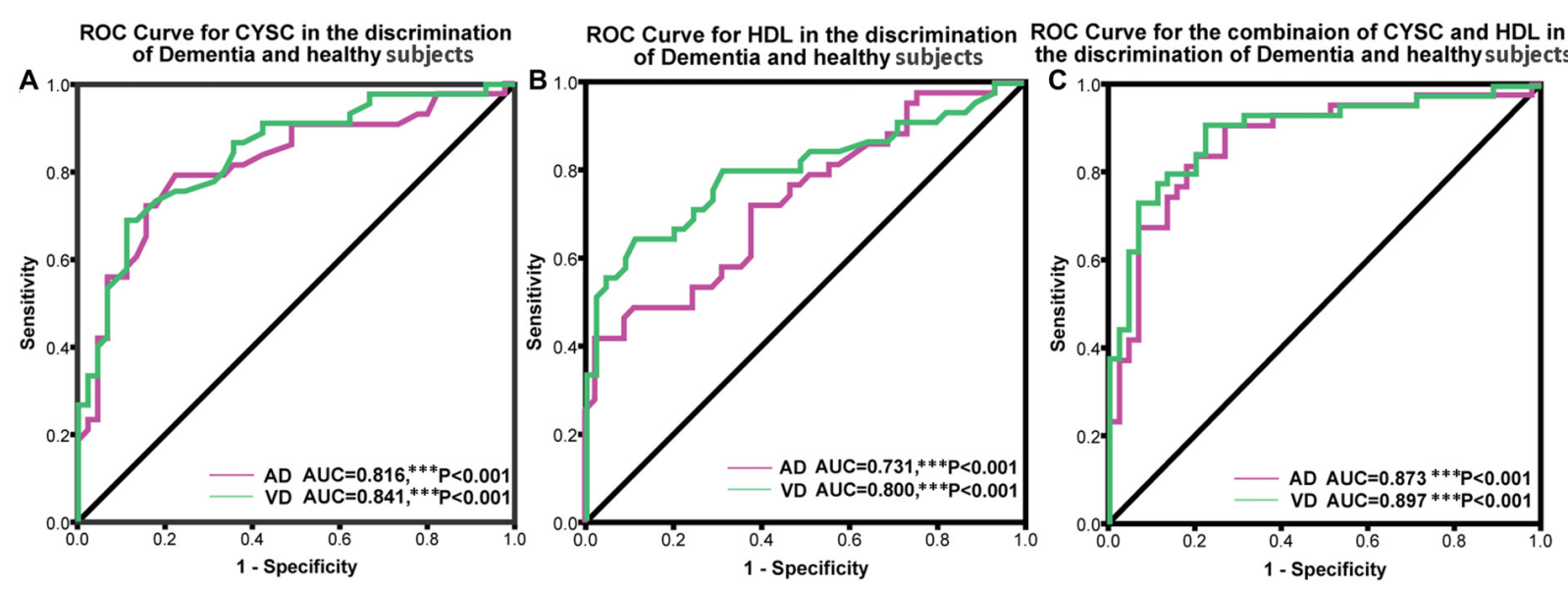

FIGURE 3 | Receiver operating characteristic (ROC) curves evaluating the utility of plasma levels of Cys $C$ and HDL in distinguishing patients with dementia from healthy controls. (A) The area under the curves (AUCs) of the ROC curves for Cys C in AD and VaD were 0.816 (95\% Cl: 0.724-0.908, ${ }^{* * *} p<0.001$ ) and 0.841 (95\% Cl: $0.627-0.835,{ }^{* * *} p<0.001$ ). (B) The AUCs of the ROC curves for HDL in AD and VaD were 0.731 (95\% Cl: $0.759-0.923$, ${ }^{* * *} p<0.001$ ) and 0.800 (95\% Cl: $\left.0.705-0.894,{ }^{* * *} p<0.001\right)$. (C) The AUCs of the ROC curves for Cys C + HDL in AD and VaD were $0.873(95 \% \mathrm{Cl}$ : $\left.0.797-0.950,{ }^{* * *} p<0.001\right)$ and 0.897 (95\% Cl: 0.831-0.964, $\left.{ }^{* * *} p<0.001\right)$.

Zhong et al., 2013). Sundelöf et al. (2008) also indicated that low levels of plasma Cys C precede clinical AD in elderly men who are free of dementia at baseline and may be an indicator of future $\mathrm{AD}$ risk. We speculate that during the early stages of diseases such as MCI, Cys C fails to protect neurons from the toxicity of oligomeric $A \beta$, which contributes to $\mathrm{AD}$ (Kaur and Levy, 2012), while during the later stages of dementia, Cys $C$ levels increase in response to disease progression to protect neurons from further damage. Differences in study design and a lack of correlation between CSF Cys C and plasma Cys C levels or GFR may explain the differences in the results of studies regarding Cys $\mathrm{C}$ and $\mathrm{AD}$ risk. Prospective studies regarding Cys $\mathrm{C}$ levels in CSF in relation to future $\mathrm{AD}$ risk are lacking. Thus, longitudinal studies of Cys $\mathrm{C}$ levels in CSF in relation to AD risk are needed (Sundelöf et al., 2010).

Low levels of HDL were associated with an increased risk for neuronal degeneration (Ward et al., 2010; Liu et al., 2014; Cai et al., 2016; Zhang et al., 2016). We noted decreased plasma HDL levels in $\mathrm{AD}$ and $\mathrm{VaD}$ patients compared to healthy subjects, implying that $\mathrm{HDL}$ contributes to the pathophysiological mechanisms underlying these disease processes. HDL-mediated anti-oxidative stress processes play an important role in the pathogenesis of $\mathrm{AD}$ and $\mathrm{VaD}$. The precise mechanisms underlying the involvement of $\mathrm{HDL}$ in $\mathrm{AD}$ and $\mathrm{VaD}$ have not yet been elucidated, but we theorize that decreases in plasma HDL may exert vasoprotective effects, preserve cognitive function, and weaken anti-inflammatory responses in $\mathrm{AD}$ and $\mathrm{VaD}$ patients (Patterson and Holahan, 2012; Hottman et al., 2014; Stukas et al., 2014). Cys C and HDL are strongly correlated with vascular function (Kaur and Levy, 2012; Dias et al., 2014; Hottman et al., 2014; Villeneuve et al., 2014). Here, we observed that the plasma levels of Cys $\mathrm{C}$ and HDL were higher and lower, respectively, in $\mathrm{VaD}$ than in $\mathrm{AD}$, respectively. This strongly suggests that vascular remodeling plays a more important role in $\mathrm{VaD}$ than in AD (Kaur and Levy, 2012; Dias et al., 2014; Villeneuve et al., 2014). 
Numerous lines of evidence indicate that Cys C and HDL are associated with cognitive diseases (Kaur and Levy, 2012). In this study, we observed correlations between Cys C levels and Hachinski scores and age via Pearson's correlation analysis. After dividing the subjects according to gender, we observed a positive correlation between IADL/MMSE sores and plasma Cys $\mathrm{C}$ levels in female AD patients. These results strongly imply that plasma Cys $\mathrm{C}$ levels can be used to assess cognitive dysfunction and quality of life in patients with dementia.

Because multiple lines of evidence support the neuroprotective roles of Cys $\mathrm{C}$ and $\mathrm{HDL}$ in neurodegenerative disorders and cognitive decline (Ghidoni et al., 2011; Hottman et al., 2014), we aimed to determine whether the combination of Cys $\mathrm{C}$ and $\mathrm{HDL}$ levels can be applied to distinguish $\mathrm{AD}$ and $\mathrm{VaD}$ patients from healthy subjects and to evaluate disease progression. To determine the diagnostic value of plasma Cys $\mathrm{C}$ and $\mathrm{HDL}$ in $\mathrm{AD}$ and $\mathrm{VaD}$, we conducted an ROC analysis and noted an AUC of 0.816 for Cys C and an AUC of 0.731 for HDL in AD and an AUC of 0.841 for Cys C and an AUC of 0.80 for $\mathrm{HDL}$ in $\mathrm{VaD}$. The AUCs for Cys $\mathrm{C}$ and $\mathrm{HDL}$ were all higher than 0.70 in $\mathrm{AD}$ and $\mathrm{VaD}$, which means that both Cys $\mathrm{C}$ and HDL have high diagnostic value and an acceptable sensitivity and specificity for distinguishing $\mathrm{AD} / \mathrm{VaD}$ patients from healthy subjects. Notably, Cys C and HDL were more reliable in distinguishing patients from healthy subjects in $\mathrm{VaD}$ than in $\mathrm{AD}$ (Figures $\mathbf{3 A} \mathbf{A} \mathbf{B}$ ). Moreover, the combination of Cys $\mathrm{C}$ and $\mathrm{HDL}$ exhibited a better ability to distinguish between patients and controls than Cys $\mathrm{C}$ or HDL alone, with an AUC of 0.873 for $\mathrm{AD}$ and an AUC of 0.897 for $\mathrm{VaD}$. The reliability and potential utility of the combination of Cys $\mathrm{C}$ and HDL as a diagnostic plasma biomarker in screening for $\mathrm{AD}$ and $\mathrm{VaD}$ is $\mathrm{r}$ eflected in our ROC analysis. Our findings have important clinical relevance. Using plasma Cys $\mathrm{C}$ and HDL levels as a screening tool, clinicians may be able to detect $\mathrm{AD}$ and $\mathrm{VaD}$ and screen for early disease in $\mathrm{AD} / \mathrm{VaD}$ patients.

There were several limitations to our study that should not be ignored: (1) a small number of participants (43 AD patients, $45 \mathrm{VaD}$ patients and 45 normal subjects) were recruited; (2) genetic factors, such as the CST3 and APOA1-2 genotypes, and anti-lipemic administration were not considered in this study; and (3) to validate and complete the questionnaire, we chose only $\mathrm{AD}$ and $\mathrm{VaD}$ patients with sufficient cognitive ability, which significantly narrowed the study population. The above limitations may have resulted in bias with respect to Cys $\mathrm{C}$ and $\mathrm{HDL}$ levels in $\mathrm{AD}$ and $\mathrm{VaD}$ patients. Therefore, it is necessary to conduct larger population studies in the future.

\section{REFERENCES}

Ahmed, R. M., MacMillan, M., Bartley, L., Halliday, G. M., Kiernan, M. C., Hodges, J. R., et al. (2014). Systemic metabolism in frontotemporal dementia. Neurology 83, 1812-1818. doi: 10.1212/WNL.0000000000 000993

Bourdenx, M., Koulakiotis, N. S., Sanoudou, D., Bezard, E., Dehay, B., and Tsarbopoulos, A. (2015). Protein aggregation and neurodegeneration in prototypical neurodegenerative diseases: examples of amyloidopathies,
In summary, the findings of the current study support the idea that inflammation and vascular burden may contribute to the pathogenesis of $\mathrm{AD}$ and $\mathrm{VaD}$. Of the biomarkers studied (Creatinine $(\mathrm{Cr})$, blood urea nitrogen (BUN), UA, Cys $\mathrm{C}$ and $\mathrm{HDL}$ ), Cys $\mathrm{C}$ and $\mathrm{HDL}$ were the most suitable and reliable anti-inflammatory mediators in distinguishing $\mathrm{AD}$ and $\mathrm{VaD}$ patients from healthy subjects and in evaluating disease progression. We speculate that higher Cys $\mathrm{C}$ levels and lower HDL levels may be associated with an elevated risk of dementia and may predispose patients to progressive disease. The anti-inflammatory effects of Cys C and HDL are important as they provide testable parameters that are related to the pathophysiology of $\mathrm{AD}$ and $\mathrm{VaD}$. ROC curves, in combination with plasma Cys $\mathrm{C}$ and $\mathrm{HDL}$ levels, may be valuable for early diagnosis of $\mathrm{AD}$ and $\mathrm{VaD}$ and may be used to increase diagnostic accuracy with respect to differentiating $\mathrm{AD}$ and $\mathrm{VaD}$ patients from healthy subjects. Our findings support the use of plasma Cys $\mathrm{C}$ and HDL levels to diagnose $\mathrm{AD}$ and $\mathrm{VaD}$, but neuropathological correlations may be required to confirm the diagnoses. To our knowledge, this is the first study to consider the combination of plasma Cys $\mathrm{C}$ and HDL levels in diagnosing and assessing the severity of $\mathrm{AD}$ and $\mathrm{VaD}$. Additional studies are needed to determine whether plasma Cys $\mathrm{C}$ and HDL abnormalities are reliable parameters in distinguishing patients with dementia from healthy subjects, particularly during the early stages of disease.

\section{AUTHOR CONTRIBUTIONS}

RWang, ZC, YF, XW, JL, KJ and QW conceived and designed the clinical study; RWang, ZC, XW, YF, JL, JZ, XL, YX and QW performed the clinical study; RWang, JL, XY, ST, RWeng, $\mathrm{BH}$ and QW analyzed the data; $\mathrm{BH}$ and XL contributed reagents/materials/analysis tools; RWang, ZC, CM, KJ and QW wrote the article.

\section{ACKNOWLEDGMENTS}

This work was supported by the National Natural Science Foundation of China (Grant No. 81271427, 81471291), Science and Technology Program of Guangdong of China (2016A050502019), Natural Science Foundations of Guangdong of China (2014A020212068), Guangdong Province Key Laboratory of Brain Function and Disease Foundation, Fundamental Research Funds for the Central Universities (16ykjc22), and Scientific Research Foundation of Guangzhou (2014J4100210) to QW.

tauopathies and synucleinopathies. Prog. Neurobiol. doi: 10.1016/j.pneurobio. 2015.07. 003 [Epub ahead of print].

Boyle, P. A., Yu, L., Wilson, R. S., Schneider, J. A., and Bennett, D. A. (2013). Relation of neuropathology with cognitive decline among older persons without dementia. Front. Aging Neurosci. 5:50. doi: 10.3389/fnagi.2013.00050

Cai, R., Han, J., Sun, J., Huang, R., Tian, S., Shen, Y., et al. (2016). Plasma clusterin and the CLU gene rs11136000 variant are associated with mild cognitive impairment in type 2 diabetic patients. Front. Aging Neurosci. 8:179. doi: $10.3389 /$ fnagi.2016.00179 
Chen, D., Wei, X., Zou, J., Wang, R., Liu, X., Xu, X., et al. (2015). Contradirectional expression of serum homocysteine and uric acid as important biomarkers of multiple system atrophy severity: a cross-sectional study. Front. Cell. Neurosci. 9:247. doi: 10.3389/fncel.2015.00247

Dias, I. H., Polidori, M. C., Li, L., Weber, D., Stahl, W., Nelles, G., et al. (2014). Plasma levels of HDL and carotenoids are lower in dementia patients with vascular comorbidities. J. Alzheimers Dis. 40, 399-408. doi: 10.3233/JAD131964

Dutta, G., Barber, D. S., Zhang, P., Doperalski, N. J., and Liu, B. (2012). Involvement of dopaminergic neuronal cystatin $\mathrm{C}$ in neuronal injuryinduced microglial activation and neurotoxicity. J. Neurochem. 122, 752-763. doi: 10.1111/j.1471-4159.2012.07826.x

Gao, H., Wei, X., Liao, J., Wang, R., Xu, J., Liu, X., et al. (2015). Lower bone mineral density in patients with Parkinson's disease: a cross-sectional study from Chinese Mainland. Front. Aging Neurosci. 7:203. doi: 10.3389/fnagi.2015. 00203

Gauthier, S., Kaur, G., Mi, W., Tizon, B., and Levy, E. (2011). Protective mechanisms by cystatin $\mathrm{C}$ in neurodegenerative diseases. Front. Biosci. (Schol. Ed.) 3, 541-554. doi: 10.2741/s170

Ghidoni, R., Benussi, L., Glionna, M., Desenzani, S., Albertini, V., Levy, E., et al. (2010). Plasma cystatin C and risk of developing Alzheimer's disease in subjects with mild cognitive impairment. J. Alzheimers Dis. 22, 985-991. doi: 10.3233/JAD-2010-101095

Ghidoni, R., Paterlini, A., Albertini, V., Glionna, M., Monti, E., Schiaffonati, L., et al. (2011). Cystatin C is released in association with exosomes: a new tool of neuronal communication which is unbalanced in Alzheimer's disease. Neurobiol. Aging 32, 1435-1442. doi: 10.1016/j.neurobiolaging.2009. 08.013

Hansson, S. F., Andréasson, U., Wall, M., Skoog, I., Andreasen, N., Wallin, A., et al. (2009). Reduced levels of amyloid- $\beta$-binding proteins in cerebrospinal fluid from Alzheimer's disease patients. J. Alzheimers Dis. 16, 389-397. doi: 10.3233/JAD-2009-0966

Heywood, W. E., Galimberti, D., Bliss, E., Sirka, E., Paterson, R. W., Magdalinou, N. K., et al. (2015). Identification of novel CSF biomarkers for neurodegeneration and their validation by a high-throughput multiplexed targeted proteomic assay. Mol. Neurodegener. 10:64. doi: 10.1186/s13024-0150059-y

Hoppe, J. B., Salbego, C. G., and Cimarosti, H. (2015). SUMOylation: novel neuroprotective approach for Alzheimer's disease? Aging Dis. 6, 322-330. doi: 10.14336/AD.2014.1205

Hottman, D. A., Chernick, D., Cheng, S., Wang, Z., and Li, L. (2014). HDL and cognition in neurodegenerative disorders. Neurobiol. Dis. 72, 22-36. doi: $10.1016 /$ j.nbd.2014.07.015

Jonsdottir, G., Ingolfsdottir, I. E., Thormodsson, F. R., and Petersen, P. H. (2013). Endogenous aggregates of amyloidogenic cystatin $\mathrm{C}$ variant are removed by THP-1 cells in vitro and induce differentiation and a proinflammatory response. Neurobiol. Aging 34, 1389-1396. doi: 10.1016/j.neurobiolaging.2012. 11.012

Kandimalla, R. J., Anand, R., Veeramanikandan, R., Wani, W. Y., Prabhakar, S., Grover, V. K., et al. (2014). CSF ubiquitin as a specific biomarker in Alzheimer's disease. Curr. Alzheimer Res. 11, 340-348. doi: 10.2174/1567205011666140331161027

Kandimalla, R. J., Prabhakar, S., Binukumar, B. K., Wani, W. Y., Gupta, N., Sharma, D. R., et al. (2011). Apo-E€4 allele in conjunction with A $\beta 42$ and tau in CSF: biomarker for Alzheimer's disease. Curr. Alzheimer Res. 8, 187-196. doi: 10.2174/156720511795256071

Kaufman, E., Hall, S., Surova, Y., Widner, H. K., Hansson, O., and Lindqvist, D. (2013). Proinflammatory cytokines are elevated in serum of patients with multiple system atrophy. PLoS One 8:e62354. doi: 10.1371/journal.pone. 0062354

Kaur, G., and Levy, E. (2012). Cystatin C in Alzheimer's disease. Front. Mol. Neurosci. 5:79. doi: 10.3389/fnmol.2012.00079

Kaur, G., Mohan, P., Pawlik, M., DeRosa, S., Fajiculay, J., Che, S., et al. (2010). Cystatin $\mathrm{C}$ rescues degenerating neurons in a cystatin B-knockout mouse model of progressive myoclonus epilepsy. Am. J. Pathol. 177, 2256-2267. doi: 10.2353/ajpath.2010.100461

Liu, Y., Li, J., Wang, Z., Yu, Z., and Chen, G. (2014). Attenuation of early brain injury and learning deficits following experimental subarachnoid hemorrhage secondary to Cystatin C: possible involvement of the autophagy pathway. Mol. Neurobiol. 49, 1043-1054. doi: 10.1007/s12035-0138579-3

McKhann, G., Drachman, D., Folstein, M., Katzman, R., Price, D., and Stadlan, E. M. (1984). Clinical diagnosis of Alzheimer's disease: report of the NINCDS-ADRDA work group under the auspices of department of health and human services task force on Alzheimer's disease. Neurology 34, 939-944. doi: 10.1212/wnl.34.7.939

Mi, W., Pawlik, M., Sastre, M., Jung, S. S., Radvinsky, D. S., Klein, A. M., et al. (2007). Cystatin C inhibits amyloid- $\beta$ deposition in Alzheimer's disease mouse models. Nat. Genet. 39, 1440-1442. doi: 10.1038/ng. 2007.29

Patterson, Z. R., and Holahan, M. R. (2012). Understanding the neuroinflammatory response following concussion to develop treatment strategies. Front. Cell. Neurosci. 6:58. doi: 10.3389/fncel.2012. 00058

Paulsen, J. S., Nance, M., Kim, J. I., Carlozzi, N. E., Panegyres, P. K., Erwin, C., et al. (2013). A review of quality of life after predictive testing for and earlier identification of neurodegenerative diseases. Prog. Neurobiol. 110, 2-28. doi: 10.1016/j.pneurobio.2013.08.003

Posada-Duque, R. A., Barreto, G. E., and Cardona-Gomez, G. P. (2014). Protection after stroke: cellular effectors of neurovascular unit integrity. Front. Cell. Neurosci. 8:231. doi: 10.3389/fncel.2014.00231

Ray, L., Khemka, V. K., Behera, P., Bandyopadhyay, K., Pal, S., Pal, K., et al. (2013). Serum homocysteine, dehydroepiandrosterone sulphate and lipoprotein (a) in Alzheimer's disease and vascular dementia. Aging Dis. 4, 57-64.

Román, G. C., Tatemichi, T. K., Erkinjuntti, T., Cummings, J. L., Masdeu, J. C., Garcia, J. H., et al. (1993). Vascular dementia: diagnostic criteria for research studies. Report of the NINDS-AIREN international workshop. Neurology 43, 250-260. doi: 10.1212/WNL.43.2.250

Stukas, S., Robert, J., and Wellington, C. L. (2014). High-density lipoproteins and cerebrovascular integrity in Alzheimer's disease. Cell Metab. 19, 574-591. doi: 10.1016/j.cmet.2014.01.003

Sundelöf, J., Arnlöv, J., Ingelsson, E., Sundstrom, J., Basu, S., Zethelius, B., et al. (2008). Serum cystatin C and the risk of Alzheimer disease in elderly men. Neurology 71, 1072-1079. doi: 10.1212/01.wnl.0000326894. 40353.93

Sundelöf, J., Sundström, J., Hansson, O., Eriksdotter-Jönhagen, M., Giedraitis, V., Larsson, A., et al. (2010). Cystatin C levels are positively correlated with both $\mathrm{A} \beta_{42}$ and tau levels in cerebrospinal fluid in persons with Alzheimer's disease, mild cognitive impairment and healthy controls. J. Alzheimers Dis. 21, 471-478. doi: 10.3233/JAD-2010-091594

Villeneuve, S., Reed, B. R., Madison, C. M., Wirth, M., Marchant, N. L., Kriger, S., et al. (2014). Vascular risk and $\mathrm{A} \beta$ interact to reduce cortical thickness in AD vulnerable brain regions. Neurology 83, 40-47. doi: 10.1212/WNL. 0000000000000550

Ward, M. A., Bendlin, B. B., McLaren, D. G., Hess, T. M., Gallagher, C. L., Kastman, E. K., et al. (2010). Low HDL cholesterol is associated with lower gray matter volume in cognitively healthy adults. Front. Aging Neurosci. 2:29. doi: 10.3389/fnagi.2010.00029

$\mathrm{Xu}$, H., Finkelstein, D. I., and Adlard, P. A. (2014). Interactions of metals and Apolipoprotein E in Alzheimer's disease. Front. Aging Neurosci. 6:121. doi: 10.3389/fnagi.2014.00121

Xu, L., Sheng, J., Tang, Z., Wu, X., Yu, Y., Guo, H., et al. (2005). Cystatin $\mathrm{C}$ prevents degeneration of rat nigral dopaminergic neurons: in vitro and in vivo studies. Neurobiol. Dis. 18, 152-165. doi: 10.1016/j.nbd.2004. 08.012

Yang, D. S., Stavrides, P., Mohan, P. S., Kaushik, S., Kumar, A., Ohno, M., et al. (2011a). Reversal of autophagy dysfunction in the TgCRND8 mouse model of Alzheimer's disease ameliorates amyloid pathologies and memory deficits. Brain 134, 258-277. doi: 10.1093/brain/awq341

Yang, D. S., Stavrides, P., Mohan, P. S., Kaushik, S., Kumar, A., Ohno, M., et al. (2011b). Therapeutic effects of remediating autophagy failure in a mouse model of Alzheimer disease by enhancing lysosomal proteolysis. Autophagy 7 , 788-789. doi: 10.4161/auto.7.7.15596

Yang, D. S., Stavrides, P., Saito, M., Kumar, A., Rodriguez-Navarro, J. A., Pawlik, M., et al. (2014). Defective macroautophagic turnover of brain lipids in the TgCRND8 Alzheimer mouse model: prevention by correcting 
lysosomal proteolytic deficits. Brain 137, 3300-3318. doi: 10.1093/brain/ awu278

Zhong, X. M., Hou, L., Luo, X. N., Shi, H. S., Hu, G. Y., He, H. B., et al. (2013). Alterations of CSF cystatin C levels and their correlations with CSF $\alpha \beta 40$ and $\alpha \beta 42$ levels in patients with Alzheimer's disease, dementia with lewy bodies and the atrophic form of general paresis. PLoS One 8:e55328. doi: 10.1371/journal. pone.0055328

Zhang, Y., Zhu, C. G., Xu, R. X., Li, S., Li, X. L., Guo, Y. L., et al. (2016). HDL subfractions and very early CAD: novel findings from untreated patients in a Chinese cohort. Sci. Rep. 6:30741. doi: 10.1038/srep 30741
Conflict of Interest Statement: The authors declare that the research was conducted in the absence of any commercial or financial relationships that could be construed as a potential conflict of interest.

Copyright (c) 2017 Wang, Chen, Fu, Wei, Liao, Liu, He, Xu, Zou, Yang, Weng, Tan, McElroy, Jin and Wang. This is an open-access article distributed under the terms of the Creative Commons Attribution License (CC BY). The use, distribution and reproduction in other forums is permitted, provided the original author(s) or licensor are credited and that the original publication in this journal is cited, in accordance with accepted academic practice. No use, distribution or reproduction is permitted which does not comply with these terms. 